\title{
Facioscapulohumeral muscular dystrophy: the molecular signaling pathway
}

\begin{abstract}
Facioscapulohumeral muscular dystrophy (FSHD) is the third most common myopathy found in adults. It is a unique genetic disease because the activation of the normally inactive gene leads to the development of pathology. The molecular mechanisms and the signaling pathways remain poorly studied so far. Here the literature data is summarized and the molecular signaling pathway of FSHD is reconstructed.
\end{abstract}

Keywords: facioscapulohumeral muscular dystrophy, fshd, dux4, signaling pathways
Volume 6 Issue 5 - 2017

\author{
Eugene Klimov ${ }^{1,2}$ \\ Department of Genetics, Faculty of Biology of Lomonosov \\ Moscow State University, Russia \\ ${ }^{2}$ University diagnostic laboratory, Russia
}

\begin{abstract}
Correspondence: Eugene Klimov, Department of Genetics, Faculty of Biology of Lomonosov Moscow State University, I 19234, Moscow, Lenin Hills, I-I2, Russia,

Email klimov_eugeney@mail.ru
\end{abstract}

Received: February 14, 2017 | Published: May 05, 2017

\section{Abbreviations: FSHD, facioscapulohumeral muscular dystrophy}

\section{Introduction}

Facioscapulohumeral muscular dystrophy (FSHD) is the third most common myopathy found in adults, with an overall incidence of more than 1:20000 (source: Orphanet). It is classified among progressive muscular dystrophies, characterized by muscular fiber necrosis and degeneration giving rise to progressive muscular weakness and atrophy. Weakness usually progresses very slowly, allowing FSHD patients to adapt and compensate muscular deficiencies, at least at the functional level. ${ }^{1}$

This is the only case in which the activation of the normally inactive gene leads to the development of pathology. ${ }^{2,3}$ But molecular mechanisms of this pathology are still unclear. Here I try to summarize the literature data and reconstruct the molecular signaling pathway of FSHD.

\section{Discussion}

In most patients, FSHD is inherited as an autosomal dominant trait (FSHD1) and de novo cases are accounting for around $25 \%$ of patients. De novo cases are often in the mosaic form. Linkage studies on large families have mapped the disease locus to the subtelomeric region of chromosome 4, more specifically at 4q35-qter. This chromosomal region lacks classical genes but contains a macrosatellite repeat comprised of an array of repeated $3.3 \mathrm{~kb}$ units, named D4Z4. ${ }^{4}$ Analysis carried out in the past of a large population of healthy subjects and FSHD patients established that the number of D4Z4 repeated units on chromosome 4 varies in the general population between 11 and 110, whereas FSHD patients carry a contracted allele from 1 to 10 repeated units. ${ }^{5}$

A small proportion of patients (around 5-10\%) with features of FSHD do not harbor a contraction of the 4q35 D4Z4 array and they often have a complex pattern of inheritance - the second form of FSHD, termed FSHD2. FSHD2 patients were shown to harbor heterozygous mutations in SMCHD1 gene on chromosome 18p11.32. This gene is essential for the inactivation of the $\mathrm{X}$ chromosome (by interacting with DNA) and binds with cytosine-5-methyltransferase DNMT3B. ${ }^{6}$ Mutations apparently cause a loss of function of the protein and the pathogenesis of the disease is likely due to SMCHD1 haploin sufficiency. Therefore FSHD2 is a digenic disorder, which requires both a loss-of-function mutation in one copy of SMCHD1 and a permissive 4qA allele. The lack of activity of SMCHD1 cannot effectively set the de novo methylation of the D4Z4 locus.

To reconstruct the signaling pathways I used Pathway Studio $9{ }^{\circledR}$ software and abstract database ResNet12 ${ }^{\circledR}$ by Elsevier. ResNet12 ${ }^{\circledR}$ database contains information from literature sources freely available on the Internet, as of December, 2015. The signaling pathway of FSHD is presented on the figure. The data from experiments using biopsies, patients' cell cultures model and mouse FSHD model was used (Figure 1).

The muscle fiber necrosis and degeneration occurs due to activation of transcription of DUX4 gene. ${ }^{8}$ Genes $F R G 1$ and $F R G 2$ are adjacent to $D U X 44^{9,10} F R G 1$ activation leads to increased activity of spliceosome. Also, with use of mouse model of FSHD, it was shown that FRG1 reduces the stability of mRNA of RBFOX1, ${ }^{11}$ which plays an important role in alternative splicing - binds to exonintron recognition motifs. Thus, the splicing process is activated, but the accuracy is reduced. This apparently leads to accumulation of abnormal splicing products, which adversely affects the cell cycle. Also FRG1 positively affect the passage of the cells in G1 phase in mouse model of FSHD. ${ }^{12}$ The role of the gene product FRG2 is not clear yet.

The transcription factor SP1 is also a target of DUX4. ${ }^{13}$ Its activation is required for transcription initiation of CDKN1A, which, in turn, blocks the cell cycle at the G1 phase. Also DUX4 regulates the transcriptional activity of PITX1 gene. ${ }^{14}$ Protein PITX1 itself is a transcription factor for TP53. The increase of the TP53 expression leads to blocking of muscle cell differentiation and apoptosis. Also TP53 binds the promoter of TP53I3 gene, regulating its expression. TP53I3 itself binds to the promoter of plasma glutathione peroxidase 3 (GPX3), adjusting its transcriptional activity. GPX3 reduces oxidative stress by the degradation of $\mathrm{H}_{2} \mathrm{O}_{2}$.

Mutated DUX4 and SMCHD1 are shown in white-out style. Retinoic acid with blue highlights has reduced concentration. A detailed description is provided in the text. Legend is on figure. Designed in the Pathway Studio $9{ }^{\circledR}$ (Elsevier). This signaling pathway is built manually using published studies. 


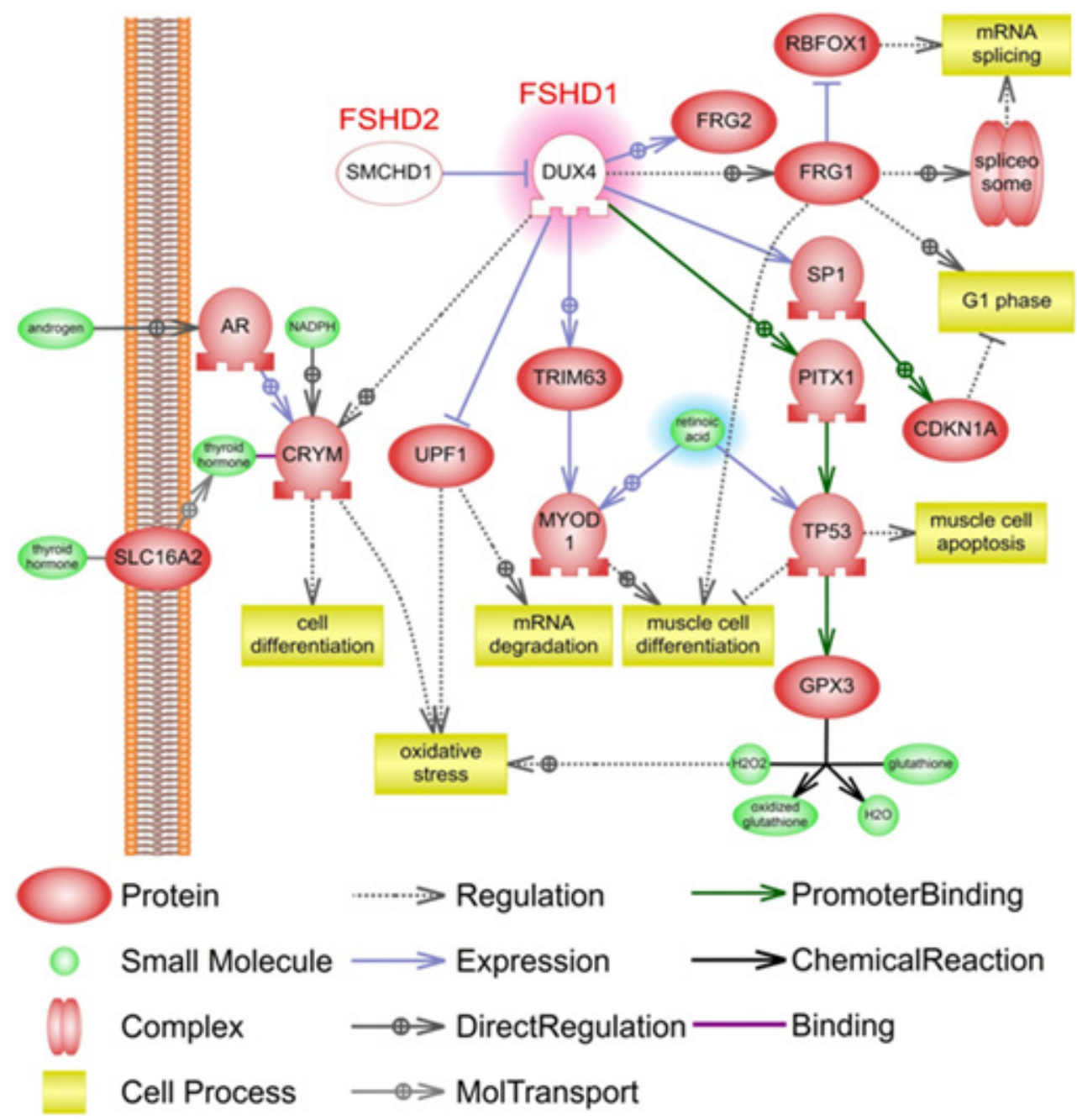

Figure I The molecular signaling pathway of facioscapulohumeral muscular dystrophy (FSHD). DUX4 with red highlights have increased expression.

TRIM63 and FBXO32 are other expression targets of DUX4. TRIM63 protein is an E3 ubiquitin ligase that localizes in the Z-line and M-line lattices of myofibrils. This protein plays an important role in the atrophy of skeletal muscle and is required for the degradation of myosin heavy chain proteins, myosin light chain, myosin binding protein, and for muscle-type creatine kinase (CKM). FBXO32 contains an F-box domain and belongs to the Fbxs class, which plays role in phosphorylation-dependent ubiquitination. This protein is highly expressed during muscle atrophy. TRIM63 enhances specifically the proteolysis of myofibrillar protein and FBXO32 contributes to reduce muscle protein synthesis. TRIM63 and FBXO32 both negatively regulate the expression of MYOD1 protein, activate its ubiquitination and turnover. This leads to blockage of muscle cell differentiation. ${ }^{15,16}$

Protein UPF1 subjected to proteolytic degradation with the participation of DUX4. ${ }^{17} \mathrm{UPF} 1$ protein itself is involved in oxidative stress response; also, UPF1 is a trigger of mRNA degradation and participates in cell differentiation. The mu-crystallin protein (CRYM) is activated in the presence of DUX4 protein. ${ }^{14}$ Mechanisms of CRYM activation are unknown. However, its direct regulators are androgen receptor (AR) and thyroid-hormone. CRYM protein influences on differentiation and oxidative stress responses. Also FSHD is characterized by a lack of retinoic acid, ${ }^{18}$ which regulates MYOD $1{ }^{19}$ and TP53. ${ }^{20}$

\section{Conclusion}

Facioscapulohumeral muscular dystrophy is the unique genetic disease. The molecular mechanisms and the signaling pathways remain poorly studied so far. With help of the mouse model of FSHD and cultured cells from patients we can better understand the molecular mechanisms of this disease, as well as the mechanisms of pathogenesis of other myodystrophies and the functioning of skeletal muscles. Meanwhile, it is now clear that the repression of DUX4 gene will block the negative signaling pathways. This repression can be carried out with the use of gene-silencing approach for example.

\section{Acknowledgments}

None.

\section{Conflicts of interest}

None.

\section{Funding}

None.

\section{References}

1. Sacconi S, Salviati L, Desnuelle C. Facioscapulohumeral muscular dystrophy. Biochimica et biophysica acta. 2015;1852(4):607-614. 
2. Lemmers RJ, van der Vliet PJ, Klooster R, et al. A unifying genetic model for facioscapulohumeral muscular dystrophy. Science. 2010;329(5999):1650-1653.

3. Mahadevan MS. Genetics. Exposing a DUX tale. Science. 2010;329(5999):1607-1608.

4. Mostacciuolo ML, Pastorello E, Vazza G, et al. Facioscapulohumeral muscular dystrophy: epidemiological and molecular study in a northeast Italian population sample. Clin Genet. 2009; 75(6):550-555.

5. van der Maarel SM, Tawil R, Tapscott SJ. Facioscapulohumeral muscular dystrophy and DUX4: breaking the silence. Trends in molecular medicine. 2011;17(5):252-258.

6. Gendrel AV, Apedaile A, Coker H, et al. Smchd1-dependent and -independent pathways determine developmental dynamics of $\mathrm{CpG}$ island methylation on the inactive X chromosome. Developmental cell. 2012;23(2):265-279.

7. Lemmers RJ, Tawil R, Petek LM, et al. Digenic inheritance of an SMCHD1 mutation and an FSHD-permissive D4Z4 allele causes facioscapulohumeral muscular dystrophy type 2. Nat Gen. 2012; 44(12):1370-1374.

8. Tassin A, Laoudj-Chenivesse D, Vanderplanck C, et al. DUX4 expression in FSHD muscle cells: how could such a rare protein cause a myopathy? J Cell Mol Med. 2013;17(1):76-89.

9. Ferri $\mathrm{G}$, Huichalaf $\mathrm{CH}$, Caccia R, et al. Direct interplay between two candidate genes in FSHD muscular dystrophy. Hum Mol Genet. 2015;24(5):1256-1266.

10. Thijssen PE, Balog J, Yao Z, et al. DUX4 promotes transcription of FRG2 by directly activating its promoter in facioscapulohumeral muscular dystrophy. Skeletal muscle. 2014;4:19.
11. Pistoni M, Shiue L, Cline MS, et al. Rbfox1 downregulation and altered calpain 3 splicing by FRG1 in a mouse model of Facioscapulohumeral muscular dystrophy (FSHD). PLoS genetics. 2013;9(1):e1003186.

12. Chen SC, Frett E, Marx J, et al. Decreased proliferation kinetics of mouse myoblasts overexpressing FRG1. PloS one. 2011;6(5):e19780.

13. $\mathrm{Xu} \mathrm{H}$, Wang Z, Jin S, et al. Dux4 induces cell cycle arrest at G1 phase through upregulation of p21 expression. Biochemical and biophysical research communications. 2014;446(1):235-240.

14. Vanderplanck C, Ansseau E, Charron S, et al. The FSHD atrophic myotube phenotype is caused by DUX4 expression. PloS one. 2011;6(10):e26820.

15. Gumucio JP, Mendias CL. Atrogin-1, MuRF-1, and sarcopenia. Endocrine. 2013;43(1):12-21.

16. Bodine SC, Baehr LM. Skeletal muscle atrophy and the E3 ubiquitin ligases MuRF1 and MAFbx/atrogin-1. American journal of physiology Endocrinology and metabolism. 2014;307(6):E469-E484.

17. Feng Q, Snider L, Jagannathan S, et al. A feedback loop between nonsensemediated decay and the retrogene DUX4 in facioscapulohumeral muscular dystrophy. eLife. 2015;4.

18. El Haddad M, Jean E, Turki A, et al. Glutathione peroxidase 3, a new retinoid target gene, is crucial for human skeletal muscle precursor cell survival. Journal of cell science. 2012;125 (Pt 24):6147-6156.

19. Hamade A, Deries M, Begemann G, et al. Retinoic acid activates myogenesis in vivo through Fgf8 signalling. Developmental biology. 2006; 289(1): 127-140.

20. Lu J, Zhang F, Yuan Y, et al. All-trans retinoic acid upregulates the expression of $\mathrm{p} 53$ via Axin and inhibits the proliferation of glioma cells. Oncology reports. 2013;29(6):2269-2274. 